\title{
Rapid GIS-based profiling of West Nile virus transmission: defining environmental factors associated with an urban- suburban outbreak in Northeast Ohio, USA
}

A. Desiree LaBeaud ${ }^{1,2}$, Ann-Marie Gorman ${ }^{3}$, Joe Koonce ${ }^{3}$, Christopher Kippes ${ }^{4}$, John McLeod $^{4}$, Joe Lynch ${ }^{4}$, Timothy Gallagher ${ }^{4}$, Charles H. King ${ }^{2}$, Anna M. Mandalakas ${ }^{1,2,4}$

${ }^{1}$ Department of Pediatrics, ${ }^{2}$ Center for Global Health and Disease, and ${ }^{3}$ Department of Biology, Case Western Reserve University, Cleveland, OH 44106, USA; ${ }^{4}$ Cuyahoga County Board of Health, Parma, OH 44130, USA

Abstract. Human West Nile virus (WNV) infection was first detected in Cuyahoga county, Ohio, USA, in 2002. During that year's extensive epidemic/epizootic among non-immune human and bird populations, the county experienced 155 cases of severe human West Nile neurological disease $($ WNND, incidence $=11.1$ cases/100,000), with 11 fatalities. Structured serosurveys indicated that $1.9 \%$, or $\sim 26,000$ of county residents (population $=1,372,303$ ) were infected that year. In early 2003, in order to better focus monitoring and control efforts, we used a geographical information system (GIS) approach and spatial statistical analysis to identify the association of environmental factors and human population structure with the observed local risk for WNV transmission. Within the varied range of urban/suburban/rural habitats across the $1186 \mathrm{~km}^{2}$ county, exploratory analysis indicated significant clustering of WNND risk in inner-ring suburbs. Subsequent discriminant factor analysis based on inputs of census and land-use/land cover data was found to effectively classify sub-areas of the county having low, medium and high WNV risk. On a 1036 ha quadrat scale of resolution, higher risk of human infection was significantly associated with higher-income areas, increased fractionation of habitat and older housing, while it was negatively associated with areas of agricultural land, wetland or forest. The areal classification of WNV transmission risk has been validated over time through detection of increased local Culex spp. mosquito density (2002-2006), and increased frequency of WNV positive mosquito pools within the medium- and high-risk quadrats. This timely working identification of the transmission scale effectively focused control interventions against newly invasive WNV in a complex North American habitat.

Keywords: encephalitis/arbovirus, West Nile virus, epidemiologic factors, cluster analysis, geographical information system.

\section{Introduction}

Since 1999, West Nile virus (WNV) has become an established seasonal cause of illness and mortality across North America (Hayes and Gubler, 2006). Both the rapid establishment of WNV in the eastern United States (CDC, 1999) and its extensive spread

Corresponding author:

A. Desiree LaBeaud

Division of Infectious Diseases, Dept. of Pediatrics

Rainbow Babies and Children's Hospital

11100 Euclid Avenue Cleveland, Ohio 44106, USA

Tel. +1 216368 5063; Fax +1 2163684825

E-mail: adl14@case.edu across North America (CDC, 2002) indicate the high susceptibility of regional ecosystems to introduction of an "exotic" pathogen such as WNV. During each of the last seven summers, WNV-related morbidity and mortality have been observed in increasing numbers among avian, human, equine and reptilian hosts, with extensive impact in terms of health costs. This experience with WNV represents a natural model for the introduction of other lethal species (e.g. Dengue or Rift Valley Fever virus) (Peters et al., 2002), and in consequence, an extensive debate has developed over the optimal means of preventing and controlling arbovirus transmission 
within North America (Roche, 2002). Beyond the immediate impact of WNV on its most susceptible vertebrate hosts, there are concerns that WNV will have an important impact on keystone species (avian raptors) with further, significant long-term effects on local ecosystems, and greater risk for transmission of rodent-borne diseases such as LaCrosse virus, hantaviruses, Ehrlichia, and Lyme disease (Weiss, 2002). For these reasons, it is now essential to better define the ecology of WNV transmission in different landscape habitats.

As an emerging pathogen, WNV spread rapidly across the United States landscape, adapting to local habitats, mosquito and bird populations in Louisiana, Illinois, Michigan and Ohio in 2002, Colorado in 2003, and Arizona and California in 2004 (O'Leary et al., 2004; Petersen and Hayes, 2004). Both WNV transmission and the observed incidence of WNV-related disease are thought to be due to many factors, including the local susceptibility of human and animal host populations (Mandalakas et al., 2005), the temporal variation of mosquito vector abundance, the impact of mosquito control efforts, local ecology, and other environmental factors (Lord and Day, 2001; Turell et al., 2001; Mans et al., 2004; Ruiz et al., 2004). Prominent among the latter are anthropogenic factors, such as housing quality and construction, local drainage, and the risk-related behaviours of susceptible human populations (Shaman et al., 2003; Ruiz et al., 2004; LaBeaud et al., 2007).

The present analysis was conducted using data for Cuyahoga county (2002 mid-year population: $1,372,303)$, located in northeast Ohio. The county includes the city of Cleveland, its older inner-ring suburbs, and many of its newer second-ring suburbs, as well as several large regional parks, and industrial and agricultural areas. During the first-year "virgin field" WNV epidemic in the summer and fall months of 2002, we observed and geocoded 221 laboratoryconfirmed cases of WNV illness in county residents, including 155 West Nile neurological disease (WNND) cases (11.1 cases/100,000 population) with 11 fatalities (Mandalakas et al., 2005, see Fig. 1).
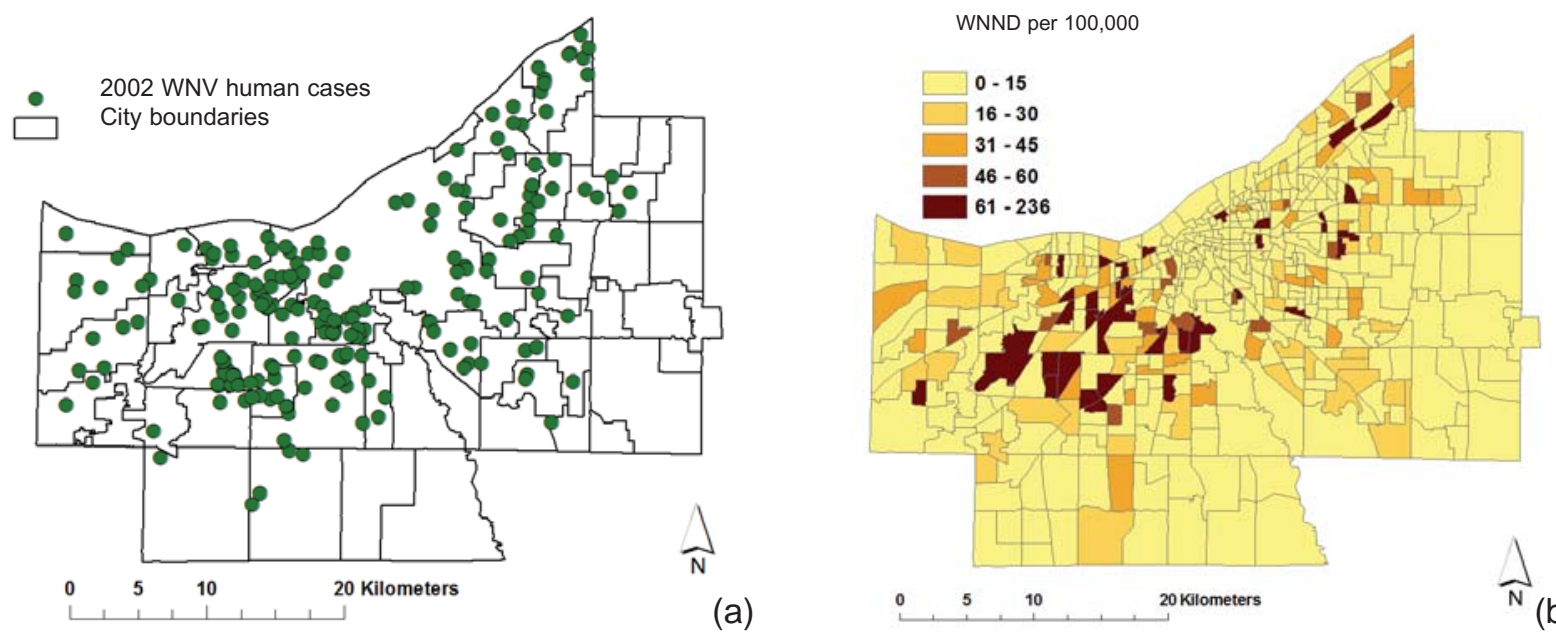

(a)

Fig. 1. (a) Distribution of severe human WNV-associated neurological disease (WNND) cases by residence location in Cuyahoga county, 2002. For this map, black outlines indicate boundaries of individual cities and municipal townships included within the county limits. To minimize the likelihood of individual cases of WNV being identified through the point maps, the geographic coordinates were offset using randomly generated vertical and horizontal distances from -100 to $100 \mathrm{~m}$. Three addresses that were initially positioned into new sampling grids as a result of this offset were placed in the appropriate grids. Offsets were applied only for visualization purposes and were not used in the spatial analyses. (b) Local age-adjusted attack rates for WNND within established US Government census tracts of Cuyahoga county. Increased age-adjusted risk is indicated by darker shades of color. For the county at-large, the 2002 rate of WNND was 16 per 100,000 population. 
The objective of the present analysis was to identify significant spatial and temporal clustering of cases within the county environment, and to link these observations to underlying environmental features that could likely contribute to transmission risk. Following risk stratification of neighbourhoods by discriminant analysis, we are able to identify a link between local geographical information system (GIS)-predicted environmental levels of risk and both the independently measured levels of mosquito vector (Culex spp.) abundance and WNV mosquito pool positivity within the same areas.

Cuyahoga county is an urban, suburban and partly rural county with unique complexity of geology and habitat for birds and mosquitoes. This habitat diversity provides a good prospect for the general study of WNV transmission in an urban and suburban setting.

\section{Materials and methods}

\section{Data geocoding and integration}

Data geocoding and integration for case locations, underlying population structure, and local environmental features, along with quadrat generation, was performed using ArcView 3.1 and ArcGIS 9 (ESRI, Redlands CA, USA). Census data were obtained from US Census Bureau internet resources (www.census.gov and available GIS files at http://arcdata.esri.com/data/tiger2000/tiger_download.cfm), and elevation and land use/land cover information from databases supplied by US Geological Survey (http://data.geocomm.com/catalog/index.html).

\section{Statistical analysis}

Initial exploratory data analysis for spatial clustering of WNV infection was performed using Clusterseer 2 software (BioMedWare, Ann Arbor, MI, USA). Clustering of WNND cases by patient's home location (adjusted for underlying population density) was assessed using Besag and Newell's scan statistic.
For the quadrat analysis of WNV transmission environment and scale, new areal grid-based subunits were developed on scales of 1,4 and 10 square miles using ArcGIS. After joining available demographic, income, housing, road density and land use data to the component county grid squares, clustered factor analysis and discriminant factor analysis (Minitab 14, State College, PA, USA) were used to examine significant associations between WNV human case density and these gridreferenced values.

Multivariable analysis of mosquito abundance and logistic analysis of WNV mosquito pool positivity were performed using SPSS (SPSS, Inc. Chicago, IL, USA) and R software. Variables were selected for multivariate logistic analysis if they were significant at the $\mathrm{P}<0.25$ level on univariate analysis, or considered biologically relevant. Model variables were trimmed in a step-wise fashion based on the Wald statistic, and eliminated if they did not significantly contribute to the model. Model variables were then evaluated for collinearity, and possible interaction with other variables. Variables that contradicted the linearity assumption were further characterized and either transformed, or dichotomized. The final model was then interpreted, including interaction terms and main effects, and checked for goodness of fit for the data.

\section{Mosquito trapping and identification}

Standard CDC miniature light traps and gravid traps were placed overnight at geocoded locations across the county during each transmission season (May-October) from 2002 through 2006. In 2002, 660 traps were placed on a non-systematic basis in areas of suspected WNV transmission (based on reports of suspected cases of human WNND, of observed bird mortality, or of high mosquito abundance). From 2003 onwards, repeated systematic trapping was performed using rotating coverage over a grid of 119 defined geographic locations distributed across the county at $\sim 3 \mathrm{~km}$ intervals. 
The number of traps sampled in 2003, 2004, 2005, and 2006, were 457, 520, 396 and 410, respectively. Mosquito species identification was performed with the aid of binary keys, and frozen specimens batched for pooled testing for WNV using RT-PCR technique (State of Ohio Department of Health Laboratories, Columbus, $\mathrm{OH}, \mathrm{USA})$.

\section{Results}

The problem: summer 2002, an extensive outbreak of WNND in Cuyahoga county, Ohio, USA

The first human cases of WNV infection in Northeast Ohio were recorded in mid-August 2002, presaging an extensive epidemic of WNND among county residents (Mandalakas et al., 2005), which lasted through mid-October, resulting in 221 confirmed cases of severe infection (Fig. 1a). As the syndrome of West Nile Fever was not readily diagnosable in this period, and was not a reportable illness in 2002, for purposes of the current analysis, only cases of WNND (median age 61 years, range $11-98$ years), geocoded to home residence, were used for analysis. This cohort was comprised of patients admitted to the 18 different hospitals within Cuyahoga county and/or reported by physicians through the established public health reporting system (CDC ArboNET Surveillance Network). For the county at large, the severe WNV case rate was 16 per 100,000 population. Using available US census data (year 2000) to derive age-adjusted WNV attack rates within census tract locations of the county, we observed an apparent aggregation of high standardized attack rates (to over 61 per 100,000) along the eastern and western borders of the city of Cleveland, and among its immediately neighboring (first ring) suburbs (Fig. 1b). Significant clustering of very high case locations, adjusted for underlying population, was noted for the same city border and suburban areas using Besag and Newell's scan statistic (Fig. 2).

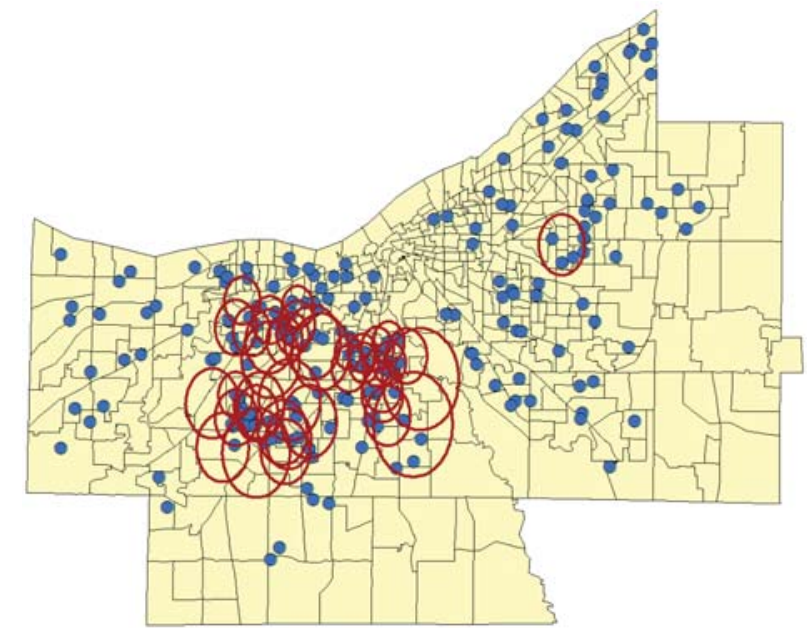

Fig. 2. Cuyahoga county census tract locations showing significant local clustering of high WNND incidence for 2002 within southern and western Cleveland and its inner ring suburbs in the west, southwest, and east. Rings indicate significant population-adjusted case clustering of four or more cases by Besag-Newell scan statistic.

\section{Landscape setting of WNV transmission}

Cuyahoga county and the city of Cleveland straddle the junction of two major land forms of North America, the glaciated Allegheny Plateau, which gradually rises eastward to the Appalachian Crest, and the Central Lowlands, which occupy the prairie areas westward into Illinois and the transMississippi plains (Fig. 3a). Its entire northern border is formed by the freshwater Lake Erie, part of the Great Lakes watershed that borders between the USA and Canada. Based on Landsat Thematic Mapper Data from October 1994, Cuyahoga county has a heterogeneous land cover with $92 \%$ of the area in three major categories $54 \%$ wooded suburban, $12 \%$ urban and $26 \%$ open urban or agricultural areas) (Fig. 3b). Since 1948, the developed land in the county has risen from $26 \%$ to $>90 \%$ of the available $1186 \mathrm{~km}^{2}$.

\section{Grid-based environmental risk assessment}

After the severe 2002 WNV transmission season, a coordinated effort was made to predict sub-regions 

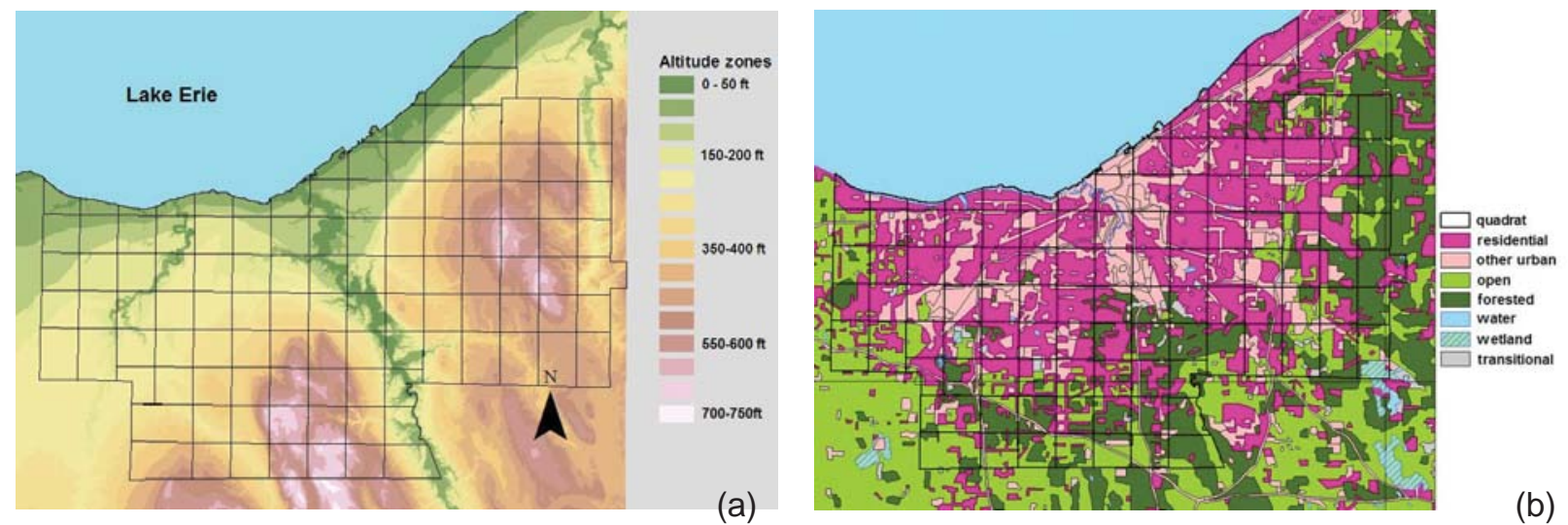

Fig. 3. Topographic and land use/land cover characteristics of Cuyahoga county. (a) Elevation map of the county, showing altitude above mean Lake Erie shoreline level. Higher altitudes in the east and south drain toward the lake to the north and west. Black outlines indicate the chosen analytic quadrat grid used in data aggregation for discriminant analysis of WNV risk; (b) land use classification of Cuyahoga county and surrounding areas. The central county is predominantly urban (magenta and pink) surrounded at the periphery by open agricultural and parkland, including forested and wetland areas.

of the county likely to have persistent high-level risk for WNV transmission in 2003 and in later years. In the risk assessment, in order to eliminate the influence of data aggregation by arbitrary political boundaries and to integrate available data on land use and habitat as well as human population, housing, and economic data into the analysis, we used GIS software to develop a new quadrat system for the county that integrated area information on gridspecified locations on scales of either 1,4 or 10 square mile grid areas. Clustered factor analysis of the association between environmental variables and grid-based WNV case density indicated a significant relationship between land-use factors (developed areas, high-intensity residential areas) as well as measures of habitat fractionation (effective land-use mesh size, or road density as a proxy of habitat fractionation) and the observed WNV case density (Table 1), particularly on the 4 square mile scale. WNV case density was greater in areas with higher income, more residents over age 65, greater road density (Fig. 4a) and older housing stock (Fig. 4b). Risk was observed to be higher in grids having a greater percentage of urban/suburban land use, and lower in areas with more parkland, agricultural use, open water or non-forested wetland.

The Cuyahoga county Board of Health had noted that in parts of the county with homes built before 1960 , catch basins located within storm sewer systems provide effective habitat for breeding and overwintering of Culex spp. mosquitoes, unless these basins were treated with larvicides. Therefore, in our next discriminate factor assessment, using three

Table 1. Unadjusted non-parametric correlation of local WNV case density with local environmental landscape features, housing, age and other human population factors within 4 square mile grid cells of Cuyahoga county.

\begin{tabular}{lrr}
\hline Variable & $\begin{array}{l}\text { Rank } \\
\text { correlation }\end{array}$ & \multicolumn{1}{c}{ P-value } \\
& 0.042 & $\mathrm{NS}^{\mathrm{a}}$ \\
\hline Total population & 0.265 & $<0.001$ \\
Population over 65 years & 0.182 & 0.002 \\
Median income & 0.131 & 0.030 \\
Median age of housing & 0.390 & $<0.001$ \\
Road density & -0.203 & 0.001 \\
Parkland density & -0.394 & $<0.001$ \\
Percent open/agricultural area & 0.513 & $<0.001$ \\
Percent urban/suburban areas & -0.218 & $<0.001$ \\
Percent open water & -0.224 & $<0.001$ \\
Percent shrub/scrub areas & -0.005 & $\mathrm{NS}^{\mathrm{a}}$ \\
Percent forest/wooded & -0.390 & $\mathrm{NS}^{\mathrm{a}}$ \\
Percent non-forested wetland & 0.017 & Comparison \\
Percent barren land & 1.00 & \\
Case density & & \\
\hline
\end{tabular}

a Not significant. 
levels of predicted grid risk (i.e. high, medium or low transmission risk/grid square) we examined whether WNV infection risk was associated with median housing age, median local income and gridcontained road length, road density, park area and park density, while adjusting for human population density and population over 65 years old. Land cover was also assessed in terms of percentage of land used for agriculture, or left as barren land, wooded versus non-forested areas, open water, shrub and scrubland or developed as urban environment. Best classification results (82\% accuracy)

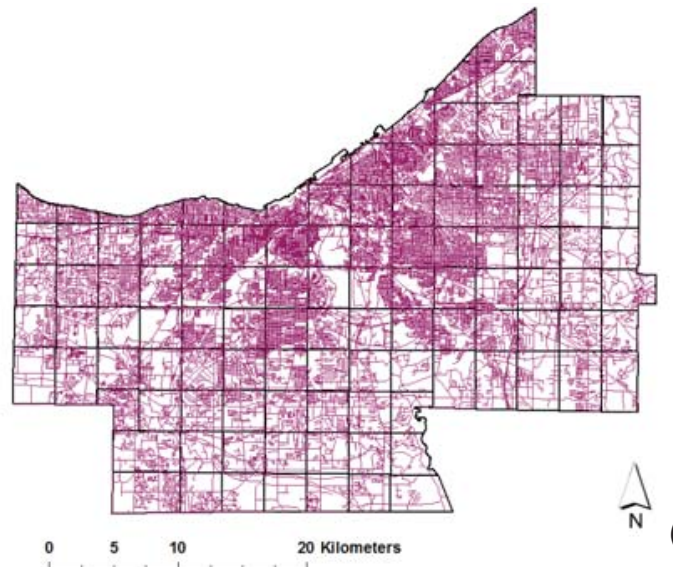

(a)

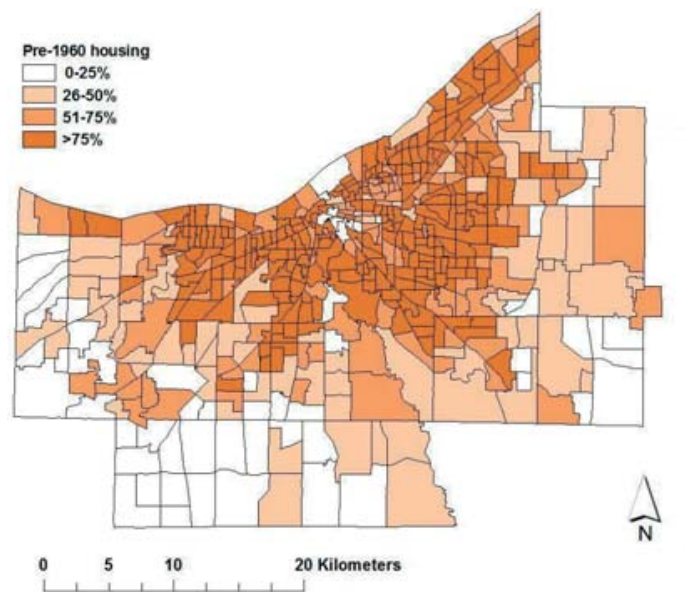

(b)

Fig. 4. Examples of GIS data inputs for risk modeling. (a) All Cuyahoga county roads in purple, with analytic quadrat grid overlaid in black; (b) location of older housing stock as determined by census tract for year 2000 US census data on age of individual domiciliary structures. were obtained using 4 square mile-gridding to define high-, intermediate- and low-risk areas within the overall region (Fig. 5). Of note, on this optimal scale of resolution, only 3 high- or medium-risk areas were misclassified as low-risk and only 3 lowrisk areas were misclassified as medium or high risk.

\section{Validating grid-based environmental risk assessment against observed mosquito outcomes}

To test whether the initial 2002 grid risk assessment, as described above, was significantly associated with other important biological factors linked to transmission, we tested whether Culex spp. mosquito abundance and WNV mosquito pool positivity were significantly different in the identified low-, mediumand high-risk grids. In order to do this, multivariable analysis was performed on mosquito trap outcomes per grid square for the period 2002-2006. Potential predictors tested in the model included individual grid risk score (low/medium/high), as possibly modified by environmental effect modifiers including trap easting (projected longitude), northing (projected latitude), shortest distance from Lake Erie, altitude, year and month of trapping.

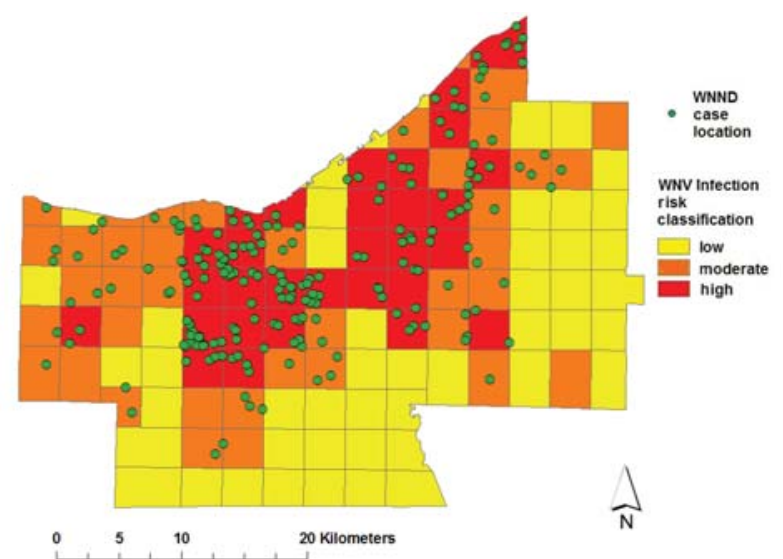

Fig. 5. Identified high-, medium- and low-risk areas for human WNV infection within Cuyahoga county in 2002, based on discriminant analysis of environmental and population features derived from US census and land-use/land-cover databases (see Figs. 3 and 4). Green circles indicate approximate residence locations of 2002 human WNND cases. 
Our analysis of 2002-2006 mosquito data indicated a significantly higher abundance of Culex spp. mosquitoes early in the season (June and July, Fig. 6a) in the high- and medium-risk areas. This phenomenon is followed in July-September by more frequent detection of WNV-positive mosquito pools in these same areas. Not shown, analysis of mosquito trap numbers by month and year revealed that the severe year for WNND, 2002, was different in that peak mosquito numbers were present for all grid squares in May of that year. In multivariable logistic analysis of gravid trap WNV positivity, there was a significant variation by month and year of collection, but even with adjustment, the grid-based risk level
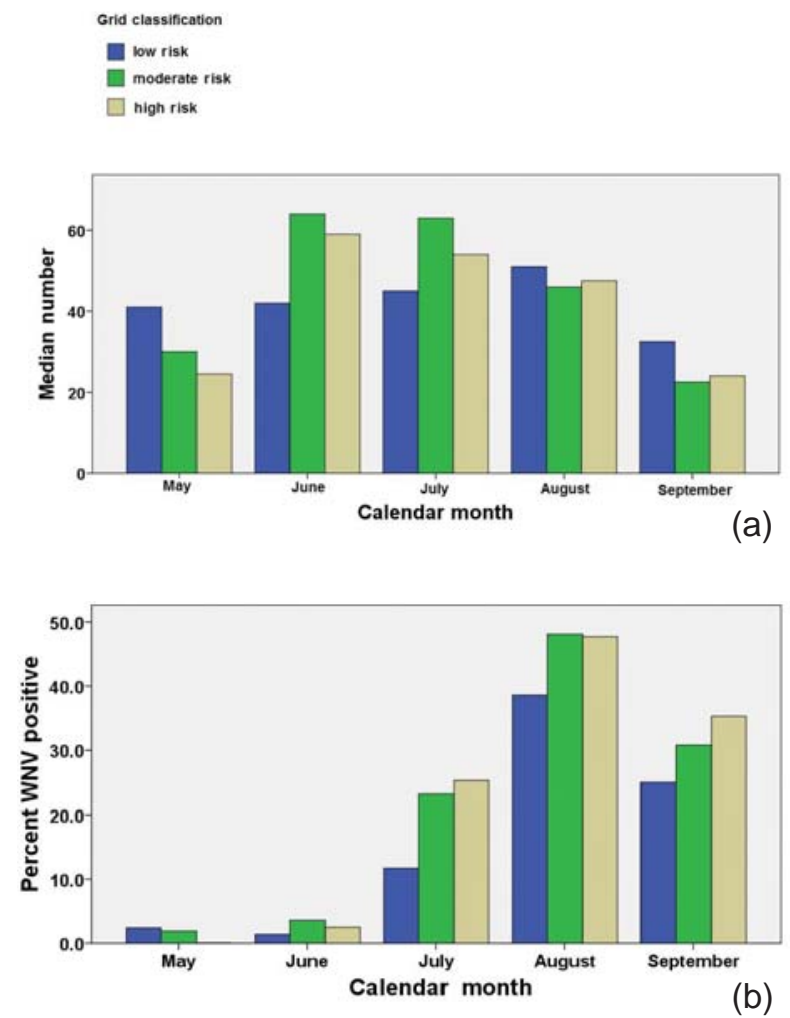

Fig. 6. Observed variation in mosquito trap outcomes according to grid area classification for WNV risk. (a) Median mosquito numbers recovered per gravid trap night in high-, moderate- and low-risk grid areas; (b) percentage of per trap mosquito pools that tested positive for WNV each month in the stratified grid risk areas between May 2002 and September 2006. assessment remained a significant predictor, with medium- and high-risk grid areas having respectively, $53 \%$ and $67 \%$, greater odds of producing a WNV positive mosquito trap result. Easting, northing, altitude and distance from water (lakes, ponds or streams) did not prove to be significant predictors in the multivariable models, and were not included in the final model presented in Table 2.

\section{Discussion}

Our results indicate that, over a $1186 \mathrm{~km}^{2}$ county control area, WNV transmission risk effectively resolves into high, medium and low risk areas on the scale of $a \sim 4$ square mile grid. As in many complex systems (Kitron et al., 2006), the ecology of WNV transmission is a function of interactions between mosquito, bird and human hosts, which

Table 2. Link between gridded landscape/population-based risk classification and observed WNV RT-PCR positivity among mosquito pools in Cuyahoga county, 2002-2006.

\begin{tabular}{|c|c|c|c|}
\hline \multicolumn{4}{|c|}{ Mosquito pool WNV positivity } \\
\hline Model variable & $\begin{array}{l}\text { Multiply-adjusted } \\
\text { odds ratio }\end{array}$ & $95 \% \mathrm{CI}^{\mathrm{a}}$ & P-value \\
\hline \multicolumn{4}{|l|}{ Calendar month } \\
\hline May & Reference & -- & -- \\
\hline June & 1.74 & $0.4-7.9$ & $N S^{b}$ \\
\hline July & 16.4 & $4.0-68.1$ & $<0.001$ \\
\hline August & 56.9 & $13.7-235.0$ & $<0.001$ \\
\hline September & 16.4 & $3.7-72.0$ & $<0.001$ \\
\hline \multicolumn{4}{|l|}{ Year } \\
\hline 2002 & 4.2 & $2.9-6.1$ & $<0.001$ \\
\hline 2004 & 0.7 & $0.5-1.0$ & $N S^{b}$ \\
\hline 2005 & 1.0 & $0.7-1.6$ & $N S^{b}$ \\
\hline 2006 & Reference & -- & -- \\
\hline
\end{tabular}

Grid risk category

\begin{tabular}{llcr} 
Low & Reference & -- & -- \\
Medium & 1.5 & $1.1-2.2$ & 0.020 \\
High & 1.7 & $1.2-2.4$ & 0.004 \\
\hline
\end{tabular}

${ }^{\mathrm{a}}$ Confidence interval; ${ }^{\mathrm{b}}$ Not significant. 
at first glance, might seem quite difficult to predict based on limited data inputs. In the case of our Cuyahoga County analysis, the strength of the GIS analysis was its ability to integrate large amounts of readily available environmental data (land use classification, human population data, as well as proxies for drainage features and habitat fractionation) in an empirical formulation of risk areas across the greater county area. This early environment-based classification proved to capture important variation of Culex spp. abundance and mosquito pool WNV positivity across the county. Furthermore, over the past 5 years, the designated areas have provided the basis for more systematic seasonal mosquito sampling, and also serve as a useful "implementation unit" for countywide mosquito abatement planning.

Over the past five decades, the geographic range of WNV has been increasing dramatically, reaching into many new habitats. Epidemics have been reported in Israel and Egypt since the 1950s (Weinberger et al., 2001), France and Italy since the 1960s, Romania in 1996-1997 (Tsai et al., 1998), and in North America in 1999-2006 (CDC, 1999). There is significant local variation in WNV transmissibility within a given enzootic area, as well as significant variation in transmission over time. In temperate northern climates, epidemics of WNV encephalitis typically occur only in the late summer and early fall. Although over 130 species of birds and 36 species of insects have been found infected with WNV, it is clear that different bird and mosquito species pose widely varying risks for transmission to humans (Goddard et al., 2002; Turell et al., 2002).

Observed WNV infection rates can be affected by a population's background immunity, practice of personal protection, mosquito vectors and their density, vector control efforts, poor housing quality, inadequate screening and local ecology (Shaman et al., 2002; Hayes and O'Leary, 2004; Ruiz et al., 2004). Greater WNND incidence should result from outbreaks in older populations, increased virus virulence, and higher WNV infection rates. In the first wave of transmission, Cuyahoga county reported $\sim 3$ times more WNND cases in 2002 than New York City (NYC) reported in 1999, although NYC population is nearly 14 times larger in size. The populations of both Cuyahoga county and NYC were immunologically naïve and exposed to higher than normal levels of urban Culex mosquitoes, Cx. pipiens and Cx. restuans (Mans et al., 2004). Although the proportion of residents aged 65 years and older differed between Cuyahoga county $(15.6 \%, 2002$ US census data) and NYC (11.7\%, 2000 US census data), this small difference is unlikely to account for the degree of difference in WNND incidence. The Cuyahoga county WNND to infection ratio was remarkably similar to the 1999 NYC (Mostashari et al., 2001) and 1996 Romania outbreaks (Tsai et al., 1998), suggesting that virus virulence did not change. In addition, recent genetic studies of WNV isolates from the United States have suggested that the genome remains remarkably stable (Lanciotti et al., 2002). Similar infection rates, no change in virulence, and a very small difference in the age structure of the two populations suggest that another explanation is needed for differences in local transmission patterns.

Ohio has been the site of numerous mosquitoborne disease outbreaks, including St. Louis encephalitis (SLE), malaria, eastern equine encephalitis and LaCrosse encephalitis, suggesting that our local ecology and environment may foster such outbreaks. Most notable, the 1975 SLE outbreak resulted in 84 cases and 8 fatalities within Cuyahoga county. Both the 1975 SLE outbreak and the 2002 WNV outbreak were preceded by mild winters, which probably allowed vector Culex mosquitoes to overwinter with lower mortality, and then propagate earlier in the season (Fig. 7). The 2002 WNV outbreak may also have been enhanced by a spring season with high precipitation, followed by a relative period of drought (Shaman et al., 2002, 2003).

Analysis of our study area, Cuyahoga county, confirms that WNV transmission was quite uneven within the irregular borders of the 59 various component municipalities, which vary from 0.1 to 78 
square miles in size. This variance within political administrative boundaries suggested the need for a more systematic assessment of the role played by focal habitat and reservoir factors in facilitating or retarding transmission. Dynamic modeling of vector-borne diseases indicates that some features of the insect-bird-human transmission cycle are likely to be more significant than others in determining risk for infection (Garrett-Jones, 1964; Dye, 1994). In particular, the vector's biting rate, its abundance relative to birds and humans, and its preference for feeding on a single versus several hosts, all have a significant impact on the observed rates of local WNV transmission. Bird diversity and abundance can increase across a gradient of rural-suburban habitats due to increase environmental fractionation (and possibly limitations on predator abundance) (Blair, 1996). Our discriminant analysis suggests that greater fractionation of habitat strongly coincides with increases in WNV transmission risk.

Not all ecological factors can be measured in sufficient detail to fully define an individual area's WNV transmission risk (Dye, 1986). Unfortunately, a recent survey of 25 different agencies responsible for directing WNV and mosquito abatement pro-

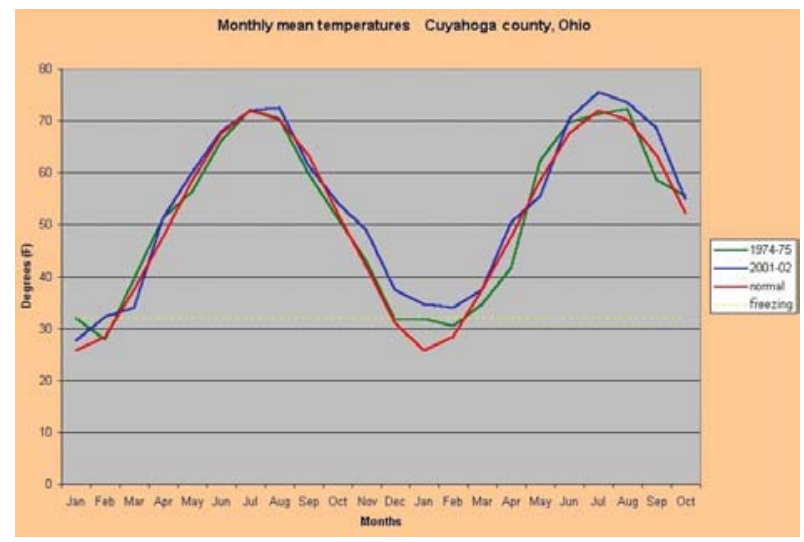

Fig. 7. Mean monthly temperatures in Cuyahoga county, Ohio (red line), showing two periods of anomalous warm winter temperatures in 1974-1975 (green line), and in 20012002 (blue line). The 1974-1975 event was associated with an outbreak of SLE virus-related disease. The 2001-2002 event was associated with the county's first WNV epidemic. grammes (Stein and Claborn, 2005) indicates that there is currently no consistency in monitoring or implementing WNV vector control across the Eastern United States. However, as we have demonstrated, within a given region it appears that dynamics of transmission self-organize on a meso-scale (1 to 5 square miles), such that risk is linked to measurable areal landscape attributes likely to be affecting local mosquito and bird populations. From the mosquito management standpoint, information on such scales can offer a useful support framework to focus intervention (Kitron et al., 2006). On this basis, we believe that it is possible to develop accurate predictions of meso-scale WNV transmission threats, providing the opportunity to design more efficient interventions for prevention of human infection and disease.

WNND case clustering and pockets of high risk identified within the county offer strong evidence that WNV risk varies on small definable scales. Because human and land use factors were able to accurately define areas in the county at high risk for WNV transmission, it is probable that these same areas will be at risk for other mosquito-borne infections. These identified transmission "hotspots" can be closely monitored for the transmission of known, endemic pathogens, such as WNV, SLE or LaCrosse virus, as well as any additional emerging mosquitoborne diseases of concern. Available public health resources can be focused on these hotspots during outbreaks. In this fashion, effective inputs on transmission heterogeneity can leverage control efforts to more effectively halt the spread of disease within regions of this size and complexity.

\section{Acknowledgements}

The authors would like to give thanks to the residents of Cuyahoga county who participated in this study and the agencies who supported this effort including the Cuyahoga county Board of Health, the Centers for Disease Control and Prevention (CDC), the Ohio Department of Health, the City of Cleveland Health Department, the City of Lakewood Health Department, the St. Luke's Foundation, the Mt. Sinai Health 
Care Foundation, the George Gund Foundation and the Sisters of Charity/St. Ann's Foundation. The authors would like to give special thanks to Mr. Terry Allan, Health Commissioner for the Cuyahoga county Board of Health, and Dr. Grant "Roy" Campbell, CDC for their invaluable insight and critical review. We would also like to thank Dr. Ken Stein for his assistance with mosquito trap analysis. This work was supported in part by NIH grants K23 HD40982 and TW/ES01543.

\section{References}

Blair RB, 1996. Land use and avian species diversity along an urban gradient. Ecol Appl 6, 506-519.

CDC, 1999. Outbreak of West Nile-like viral encephalitisNew York, 1999. Morb Mortal Wkly Rep 48, 845-849.

CDC, 2002. West Nile virus activity-United States, September 12-18, 2002, and Ohio, January 1-September 12, 2002. Morb Mortal Wkly Rep 51, 836-837.

Dye C, 1986. Vectorial capacity: must we measure all its components? Parasitol Today 2, 203-209.

Dye C, 1994. Approaches to vector control: the epidemiological context of vector control. Trans R Soc Trop Med Hyg 88, 147-149.

Garrett-Jones C, 1964. Prognosis for interruption of malaria transmission through the assessment of the mosquito's vectorial capacity. Nature 204, 1173-1175.

Goddard LB, Roth AE, Reisen WK, Scott TW, 2002. Vector competence of California mosquitoes for West Nile virus. Emerg Infect Dis 8, 1385-1391.

Hayes EB, Gubler DJ, 2006. West Nile virus: epidemiology and clinical features of an emerging epidemic in the United States. Annu Rev Med 57, 181-194.

Hayes EB, O’Leary DR, 2004. West Nile virus infection: a pediatric perspective. Pediatrics 113, 1375-1381.

Kitron U, Clennon JA, Cecere MC, Gürtler RE, King CH, Vazquez-Prokopec G, 2006. Upscale or downscale: applications of fine scale remotely sensed data to Chagas disease in Argentina and schistosomiasis in Kenya. Geospatial Health 1, 49-58.

LaBeaud AD, Kile JR, Kippes C, King CH, Mandalakas AM, 2007. Exposure to West Nile virus during the 2002 epidemic in Cuyahoga county, Ohio: a comparison of pediatric and adult behaviors. Public Health Rep 122, 356361 .
Lanciotti RS, Ebel GD, Deubel V, Kerst AJ, Murri S, Meyer R, Bowen M, McKinney N, Morrill WE, Crabtree MB, Kramer LD, Roehrig JT, 2002. Complete genome sequences and phylogenetic analysis of West Nile virus strains isolated from the United States, Europe, and the Middle East. Virology 298, 96-105.

Lord CC, Day JF, 2001. Simulation studies of St. Louis encephalitis and West Nile viruses: the impact of bird mortality. Vector Borne Zoonotic Dis 1, 317-329.

Mandalakas AM, Kippes C, Sedransk J, Kile JR, Garg A, McLeod J, Berry RL, Marfin AA, 2005. West Nile virus epidemic, northeast Ohio, 2002. Emerg Infect Dis 11, 17741777.

Mans NZ, Yurgionas SE, Garvin MC, Gary RE, Bresky JD, Galaitsis AC, Ohajuruka OA, 2004. West Nile virus in mosquitoes of Northern Ohio, 2001-2002. Am J Trop Med Hyg 70, 562-565.

Mostashari F, Bunning ML, Kitsutani PT, Singer DA, Nash D, Cooper MJ, Katz N, Liljebjelke KA, Biggerstaff BJ, Fine AD, Layton MC, Mullin SM, Johnson AJ, Martin DA, Hayes EB, Campbell GL, 2001. Epidemic West Nile encephalitis, New York, 1999: results of a household-based seroepidemiological survey. Lancet 358, 261-264.

O’Leary DR, Marfin AA, Montgomery SP, Kipp AM, Lehman JA, Biggerstaff BJ, Elko VL, Collins PD, Jones JE, Campbell GL, 2004. The epidemic of West Nile virus in the United States, 2002. Vector Borne Zoonotic Dis 4, 61-70.

Peters CJ, Spertzel R, Patrick W, 2002. Aerosol technology and biological weapons. In: Biological threats and terrorism: assessing the science and response capabilities. SL Knobler, AAF Mahmoud and LA Pray (eds). National Academy Press, USA, pp. 66-77.

Petersen LR, Hayes EB, 2004. Westward ho?-The spread of West Nile virus. N Engl J Med 351, 2257-2259.

Roche JP, 2002. Print media coverage of risk-risk tradeoffs associated with West Nile encephalitis and pesticide spraying. J Urban Health 79, 482-490.

Ruiz MO, Tedesco C, McTighe TJ, Austin C, Kitron U, 2004. Environmental and social determinants of human risk during a West Nile virus outbreak in the greater Chicago area, 2002. Int J Health Geogr 3, 8.

Shaman J, Day JF, Stieglitz M, 2002. Drought-induced amplification of Saint Louis encephalitis virus, Florida. Emerg Infect Dis $8,575-580$. 
Shaman J, Day JF, Stieglitz M, 2003. St. Louis encephalitis virus in wild birds during the 1990 south Florida epidemic: the importance of drought, wetting conditions, and the emergence of Culex nigripalpus (Diptera: Culicidae) to arboviral amplification and transmission. J Med Entomol 40, 547-554.

Stein KJ, Claborn DM, 2005. Telephonic survey of surveillance and control procedures for the mosquito vectors of West Nile virus near naval installations in the Eastern United States. Mil Med 170, 658-662.

Tsai TF, Popovici F, Cernescu C, Campbell GL, Nedelcu NI, 1998. West Nile encephalitis epidemic in southeastern Romania. Lancet 352, 767-771.
Turell MJ, O’Guinn ML, Dohm DJ, Jones JW, 2001. Vector competence of North American mosquitoes (Diptera: Culicidae) for West Nile virus. J Med Entomol 38, 130-134. Turell MJ, Sardelis MR, O’Guinn ML, Dohm DJ, 2002. Potential vectors of West Nile virus in North America. Curr Top Microbiol Immunol 267, 241-252.

Weinberger M, Pitlik SD, Gandacu D, Lang R, Nassar F, Ben David D, Rubinstein E, Izthaki A, Mishal J, Kitzes R, Siegman-Igra Y, Giladi M, Pick N, Mendelson E, Bin H, Shohat T, 2001. West Nile fever outbreak, Israel, 2000: epidemiologic aspects. Emerg Infect Dis 7, 686-691.

Weiss R, 2002. Ecological impact of West Nile Virus. Washington Post, Washington DC, USA. 